\title{
A Geometrical Treatment for Obtaining Necessary and Sufficient Conditions for Joint Quadratic Lyapunov Function Existence for State-Dependent, Switched Systems: A Two-Dimensional Case
}

\author{
Wynita M. Griggs, Christopher K. King, Robert N. Shorten, Oliver Mason and Kai Wulff
}

\begin{abstract}
The question of existence of joint quadratic Lyapunov functions (QLFs) for state-dependent, switched dynamical systems is given a preliminary geometrical treatment in this paper. The joint QLF problem for a switched system and a collection of regions defined by state vectors that determine when switching occurs consists of finding nonempty intersections of convex sets of QLFs. The existence of a joint QLF guarantees switched system stability. Necessary and sufficient conditions for the existence of a joint QLF are obtained for a two-dimensional problem.
\end{abstract}

\section{INTRODUCTION}

A great interest in stability problems arising from the study of switched and hybrid systems has arisen in recent years [1]-[5]. The study of stability for switched systems is more difficult than the study of stability for continuous systems or discrete systems due to the complicated behavior caused by interaction between continuous and discrete dynamics. Lyapunov stability theory still plays a dominating role [6], [7]. A common Lyapunov function for all subsystems comprising the switched system guarantees stability under an arbitrary switching law [3].

In certain situations, it is not necessary to guarantee stability for every possible switching signal and a number of authors have considered questions related to the stability of switched systems under restricted switching regimes. One important example of this is state-dependent switching, where the rule that determines when a switch in system dynamics may occur is determined by the value of the state vector. A genetic regulatory network in which switching occurs when the concentration of a regulatory protein crosses a threshold provides an example of this type of switching system [8]. Internet congestion control provides another example [8].

The stability problems associated with a state-dependent switching regime can loosely be divided into two categories. In the first of these, the state space is partitioned by a number of hypersurfaces that determine the mode switches in the system dynamics and the problem is to analyze the stability of the time-varying system defined in this way. In the second category of problem, one is concerned with finding statedependent rules for switching between a family of unstable

W. Griggs, R. Shorten and O. Mason are with Hamilton Institute, National University of Ireland, Maynooth, Co. Kildare, Ireland.

C. King is with Department of Mathematics, Northeastern University, 360 Huntington Avenue, Boston, MA 02115, USA.

$\mathrm{K}$. Wulff is with Technische Universität Berlin, Fachgebiet Regelungssysteme, Sekretariat EN11, Einsteinufer 17, D-10587 Berlin, Germany.

Corresponding author: wynita.griggsenuim.ie systems that result in stability. It is the former class of problem that is the topic of this paper.

Specifically, via a geometrical treatment, we address the problem of determining global asymptotic stability of a second order, state-dependent switched system with linear subsystem dynamics, given a partitioning of the state-space into closed, double conic regions that determine which of the linear subsystem components is active. Guaranteeing global asymptotic stability is achieved by finding necessary and sufficient conditions for the existence of a quadratic Lyapunov function (QLF) which is decreasing along every trajectory of the system. While second order systems are considered, it is expected that some of the algebraic results derived in this paper will prove useful in analyzing the higher-dimensional case at a later stage.

\section{Notation}

The fields of real and complex numbers are denoted by $\mathbb{R}$ and $\mathbb{C}$, respectively. Let $\mathbb{C}^{-}$denote the set of complex numbers with real part strictly less than zero (ie: $\mathbb{C}^{-}$defines the open left half of the complex plane). The set of $n \times m$ real matrices is denoted by $\mathbb{R}^{n \times m}$ and $\mathbb{R}^{n}$ denotes the set of $n \times 1$ real column vectors. Let $a_{i j}$ denote the element corresponding to the $i t h$ row and $j$ th column of a matrix $A$. A matrix $A \in \mathbb{R}^{n \times n}$ is called Hurwitz if its spectrum lies in $\mathbb{C}^{-}$. Let $\mathcal{S}_{n}(\mathbb{R})$ denote the vector space of $n \times n$ real symmetric matrices. The notations $A>0, A \geq 0$, $A<0$ and $A \leq 0$ refer to a positive definite, a positive semi-definite, a negative definite and a negative semi-definite matrix, respectively. A matrix is called positive definite if $x^{T} A x>0$ for every nonzero $x \in \mathbb{R}^{n}$. Let $\mathcal{P}_{n}(\mathbb{R})$ denote the set of $n \times n$ real symmetric positive definite matrices.

\section{BACKGROUND}

In this section, we review the QLF existence problem. We are seeking to determine global asymptotic stability of a state-dependent, switched dynamical system

$$
\dot{x}=A(x) x, A(x) \in \mathcal{A}:=\left\{A_{1}, A_{2}, \ldots, A_{N}\right\}
$$

where $A_{i}$ with $i \in\{1,2, \ldots, N\}$ are constant matrices in $\mathbb{R}^{2 \times 2}$. It is assumed that the matrices $A_{i}$ are Hurwitz. First, we recall the common quadratic Lyapunov function (CQLF) existence problem. 


\section{A. Common $Q L F$ Problem}

For a $P \in \mathcal{P}_{n}(\mathbb{R})$, the function $V(x)=x^{T} P x$ defines a QLF for the dynamical system $\dot{x}=A x$ if $P A+A^{T} P<0$. (We will henceforth often abuse notation and say that $P$ is a QLF for $A$, meaning that $V(x)$ is a QLF for $\dot{x}=A x$.) It is well known that $A$ is a Hurwitz matrix if and only if there exists a QLF for $A$ [9, Theorem 3.6]. We define the set of all such QLF matrices as

$$
\mathcal{L}(A):=\left\{P \in \mathcal{P}_{n}(\mathbb{R}) \mid P A+A^{T} P<0\right\} .
$$

Then $\mathcal{L}(A)$ is an open convex pointed cone in the space of real symmetric $n \times n$ matrices.

The CQLF existence problem involves finding conditions which guarantee the existence of a common QLF for a set of Hurwitz matrices $\left\{A_{1}, \ldots, A_{N}\right\}$. The existence of a CQLF implies stability of the dynamical system $\dot{x}=A_{\tau(t)} x$ where $\tau: \mathbb{R}^{+} \mapsto\{1, \ldots, N\}$ is any switching function. Referring to the cones defined above, an equivalent formulation is to find conditions for a nonempty intersection of the cones $\mathcal{L}\left(A_{1}\right), \ldots, \mathcal{L}\left(A_{N}\right)$. A solution to the CQLF existence problem for two-dimensional systems exists as follows (see [10] for a proof and for an extension to more than two matrices).

Theorem 1: [10] Let $A_{1}, A_{2} \in \mathbb{R}^{2 \times 2}$ be two Hurwitz matrices. A necessary and sufficient condition for the systems $\dot{x}=A_{1} x$ and $\dot{x}=A_{2} x$ to have a CQLF is that the matrices $A_{2}^{-1} A_{1}$ and $A_{2} A_{1}$ do not have real negative eigenvalues. An equivalent condition is that all convex combinations of $A_{1}$ and $A_{2}$, and of $A_{1}$ and $A_{2}^{-1}$, are Hurwitz.

\section{B. General Joint QLF Problem}

Suppose that $\Omega$ is a closed double cone in $\mathbb{R}^{n}$ such that if $x \in \Omega$ then $\lambda x \in \Omega$ for all $\lambda \in \mathbb{R}$. Given $A \in \mathbb{R}^{n \times n}$, define the QLF set for the pair $(A, \Omega)$ as follows:

$$
\begin{array}{r}
\mathcal{L}(A, \Omega):=\left\{P \in \mathcal{P}_{n}(\mathbb{R}) \mid x^{T}\left(P A+A^{T} P\right) x<0,\right. \\
\forall x \in \Omega, x \neq 0\} .
\end{array}
$$

If $A$ is Hurwitz, then $\mathcal{L}(A, \Omega)$ is nonempty. The general joint QLF problem for a collection of matrices $A_{i}$ and regions $\Omega_{i}$ is to find conditions for a nonempty intersection of the sets $\left\{\mathcal{L}\left(A_{i}, \Omega_{i}\right)\right\}$. If $\cup_{i} \Omega_{i}=\mathbb{R}^{n}$, then the existence of a joint QLF implies exponential stability of the state-dependent switching system $\dot{x}=A(x) x$, where $A(x) \in\left\{A_{1}, \ldots, A_{N}\right\}$, with $A(x)=A_{i}$ implying $x \in \Omega_{i}$.

\section{Problem Set-Up}

We aim to obtain analytical necessary and sufficient conditions for the existence of a joint QLF for the following case. Set $n=2$ and $N=2$ and let $x_{1}, x_{2}$ be two vectors in $\mathbb{R}^{2}$. Characterize $\Omega_{1}$ and $\Omega_{2}$ as follows:

$$
\Omega_{1}:=\left\{x=\alpha x_{1}+\beta x_{2} \mid \alpha \text { and } \beta \in \mathbb{R}, \alpha \beta \geq 0\right\}
$$

and let $\Omega_{2}=\mathbb{R}^{2}$. Then $\Omega_{1}$ is the set of all linear combinations of $x_{1}$ and $x_{2}$ with the restriction that the coefficients are either both non-positive or both non-negative. We will describe this region as a closed double cone in $\mathbb{R}^{2}$ defined by the vectors $x_{1}$ and $x_{2}$. The boundary of $\Omega_{1}$ is the pair of lines parallel to $x_{1}$ and $x_{2}$ passing through the origin, as depicted in Fig. 1. Given two Hurwitz matrices $A_{1}, A_{2} \in \mathbb{R}^{2 \times 2}$, $A(x) \in\left\{A_{1}, A_{2}\right\}$ may be equal to either $A_{1}$ or $A_{2}$ for all vectors $x \in \Omega_{1}$ and is equal to $A_{2}$ everywhere else. Given this setup, we are interested in determining necessary and sufficient conditions for the existence of a $P \in \mathcal{P}_{2}(\mathbb{R})$ such that the following are simultaneously satisfied:

(i) $x^{T}\left(A_{1}^{T} P+P A_{1}\right) x<0, \forall x \in \Omega_{1}, x \neq 0$;

(ii) $A_{2}^{T} P+P A_{2}<0$.

If (i) and (ii) hold, then the pairs $\left(A_{1}, \Omega_{1}\right)$ and $\left(A_{2}, \mathbb{R}^{2}\right)$ are said to have a joint QLF.

\section{EXTENSIONS OF CQLF RESULTS}

The following question arises: How does the CQLF existence problem stated in Theorem 1 translate to a joint QLF problem when one of the sets $\mathcal{L}(A)$ is replaced by a set $\mathcal{L}(A, \Omega)$ ? First, a review of the geometry of convex cones generated by Lyapunov functions is provided.

\section{A. Geometry of Convex Cones}

The boundary of $\mathcal{L}(A)$ consists of symmetric positive semi-definite matrices $P$ for which $P A+A^{T} P \leq 0$. If $P_{0}$ is in the boundary of $\mathcal{L}(A)$ and $x_{0}$ is in the kernel of $P_{0} A+A^{T} P_{0}$, then

$$
x_{0}^{T}\left(A^{T} P_{0}+P_{0} A\right) x_{0}=2 x_{0}^{T} P_{0} A x_{0}=0 .
$$

The set of all symmetric matrices $Q$ for which $x_{0}^{T} Q A x_{0}=$ 0 defines a linear subspace $H$ in the space of symmetric matrices. Since $H$ does not intersect the QLF set $\mathcal{L}(A)$ but does intersect its boundary, it follows that $H$ is tangent to $\mathcal{L}(A)$. We will view $H$ as a hyperplane in the vector space $\mathbb{R}^{3}$. This is possible because the space of real symmetric matrices is isomorphic to $\mathbb{R}^{n(n+1) / 2}$ and it is an inner product space when equipped with the Hilbert-Schmidt inner product: $\langle A, B\rangle=\operatorname{Tr} A^{T} B$. It is natural to define the hyperplane $H$ by its normal vector in $\mathbb{R}^{n(n+1) / 2}$ and to write this normal vector as a matrix. The normal is computed as follows: we

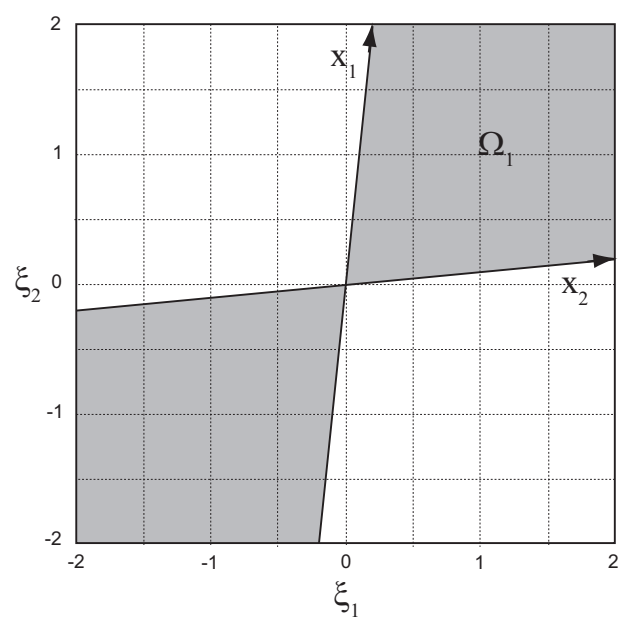

Fig. 1. Partition of the state space. 
write the equation for $H$ as

$$
\begin{aligned}
0=2 x_{0}^{T} Q A x_{0}=x_{0}^{T}(Q A & \left.+A^{T} Q\right) x_{0} \\
& =\operatorname{Tr} Q\left(A x_{0} x_{0}^{T}+x_{0} x_{0}^{T} A^{T}\right)
\end{aligned}
$$

where in the last equality we used the cyclic property of the trace. This last term precisely states that $H$ is the set of vectors $Q$ in $\mathbb{R}^{n(n+1) / 2}$ which are orthogonal to the vector $A x_{0} x_{0}^{T}+x_{0} x_{0}^{T} A^{T}$. Thus, $A x_{0} x_{0}^{T}+x_{0} x_{0}^{T} A^{T}$ is the normal vector to $H$. Furthermore, since $H$ is tangent to the set $\mathcal{L}(A)$, then this set must lie on one side of $H$; particularly, $A x_{0} x_{0}^{T}+$ $x_{0} x_{0}^{T} A^{T}$ points away from $\mathcal{L}(A)$.

For two-dimensional systems, every tangent plane to the set $\mathcal{L}(A)$ has the form described above with a normal vector of the form $A x x^{T}+x x^{T} A^{T}$ for some vector $x$ in the plane. This is because if $P$ is on the boundary of $\mathcal{L}(A)$ then $P A+A^{T} P$ must be negative semi-definite and hence in two dimensions it must have a one-dimensional kernel. Then the situation described above applies and leads to the tangent plane of the stated form. In higher dimensional systems the kernel may be more than one-dimensional, in which case other tangent planes are possible.

\section{B. Separating Tangential Hyperplane}

If $A_{1}$ and $A_{2}$ are Hurwitz matrices for which $\mathcal{L}\left(A_{1}\right)$ and $\mathcal{L}\left(A_{2}\right)$ are disjoint then $A_{1}$ and $A_{2}$ do not have a CQLF. Since $\mathcal{L}\left(A_{1}\right)$ and $\mathcal{L}\left(A_{2}\right)$ are convex sets, there is a separating hyperplane between these sets [11] and this hyperplane may be chosen to be a simultaneous tangent plane for both sets. Supposing that this tangent plane has the form described in Section III-A then there are vectors $x$ and $y$ such that the normal vector for the plane is $A_{1} x x^{T}+x x^{T} A_{1}^{T}$ at $\mathcal{L}\left(A_{1}\right)$ and $A_{2} y y^{T}+y y^{T} A_{2}^{T}$ at $\mathcal{L}\left(A_{2}\right)$. Furthermore, since the plane separates the QLF sets, these normals must be oppositely oriented, hence there is a positive constant $k$ such that

$$
A_{1} x x^{T}+x x^{T} A_{1}^{T}=-k\left(A_{2} y y^{T}+y y^{T} A_{2}^{T}\right) .
$$

Equivalently, for all symmetric matrices $Q \in \mathbb{R}^{n \times n}$, we have

$$
x^{T} Q A_{1} x=-k y^{T} Q A_{2} y .
$$

The following result allows us to solve this equation.

Lemma 2: [12] Let $x, y, u, v$ be four nonzero vectors in $\mathbb{R}^{n}$ such that for all symmetric matrices $Q \in \mathbb{R}^{n \times n}, x^{T} Q y=$ $-k u^{T} Q v$ with $k>0$. Then, either

$$
\begin{aligned}
& x=\alpha u \text { for some real scalar } \alpha \text { and } y=-\left(\frac{k}{\alpha}\right) v, \text { or } \\
& x=\beta v \text { for some real scalar } \beta \text { and } y=-\left(\frac{k}{\beta}\right) u .
\end{aligned}
$$

Refer to [12] for proof. Applying this result to (3) implies that either some convex combination of $A_{1}$ and $A_{2}$, or of $A_{1}$ and $A_{2}^{-1}$, is singular. As noted above, this conclusion applies to two-dimensional systems because every tangent plane has the required form, thereby recovering the results in [10].

\section{Extending CQLF}

Suppose that we represent symmetric matrices $Q \in \mathcal{S}_{2}(\mathbb{R})$ as points in a plane. We label the coordinates in a $\left(q_{12}, q_{22}\right)$ plane such that each point defines a symmetric matrix of the form

$$
Q=\left[\begin{array}{cc}
1 & q_{12} \\
q_{12} & q_{22}
\end{array}\right]
$$

The set of symmetric matrices whose $(1,1)$ entry is nonzero can be re-scaled to this form and matrices whose $(1,1)$ entry is zero lie in the closure of this set. Fig. 2 depicts three points and the parabola $q_{22}=q_{12}^{2}$. Points on the parabola (eg: $Q_{3}$ ) correspond to positive semi-definite matrices. Points on the positive side of the locus (eg: $Q_{1}$ ) correspond to positive definite matrices and points on the negative side of the locus (eg: $Q_{2}$ ) correspond to indefinite and negative semi-definite matrices. It is evident that the set of all positive semi-definite symmetric matrices is convex. Under the assumption that $A$ is not a triangular matrix, the projection of a set $\mathcal{L}(A)$ in this two-dimensional representation corresponds to the interior of an ellipse [10]. It is also evident that this set $\mathcal{L}(A)$ is convex.

Consider a set $\mathcal{L}(A, \Omega)$. Recall that this is the set of matrices $P \in \mathcal{P}_{2}(\mathbb{R})$ for which $x^{T} P A x<0$ for all nonzero $x \in \Omega$. It immediately follows that this set is convex and that $\mathcal{L}(A)$ is a subset of $\mathcal{L}(A, \Omega)$. It also follows that, if $x_{1}$ and $x_{2}$ define a closed double cone $\Omega$ as in Section II-C, then $\mathcal{L}(A, \Omega)$ lies between the hyperplanes $H_{1}:=$ $\left\{P \mid x_{1}^{T} P A x_{1}=0\right\}$ and $H_{2}:=\left\{P \mid x_{2}^{T} P A x_{2}=0\right\}$ and that these hyperplanes are tangent to the set. These hyperplanes define lines in the $\left(q_{12}, q_{22}\right)$-plane and examples of resulting possible configurations for $\mathcal{L}(A, \Omega)$ are shown in Figs. 3 and 4. As noted above, every tangent line to the ellipse $\mathcal{L}(A)$ has the form $\left\{P \mid x^{T} P A x=0\right\}$ for some $x \in \mathbb{R}^{2}$. As $x$ varies between $x_{1}$ and $x_{2}$, the tangent line varies from $H_{1}$ to $H_{2}$. As a consequence, any line $\left\{P \mid x^{T} P A x=0\right\}$ which is tangent to $\mathcal{L}(A)$ is also tangent to $\mathcal{L}(A, \Omega)$ when $x \in \Omega$. The following result is an extension of Theorem 1 .

Theorem 3: Let $A_{1}, A_{2} \in \mathbb{R}^{2 \times 2}$ be two Hurwitz matrices. Suppose that $\Omega_{1}$ is a closed double cone of the form (1).

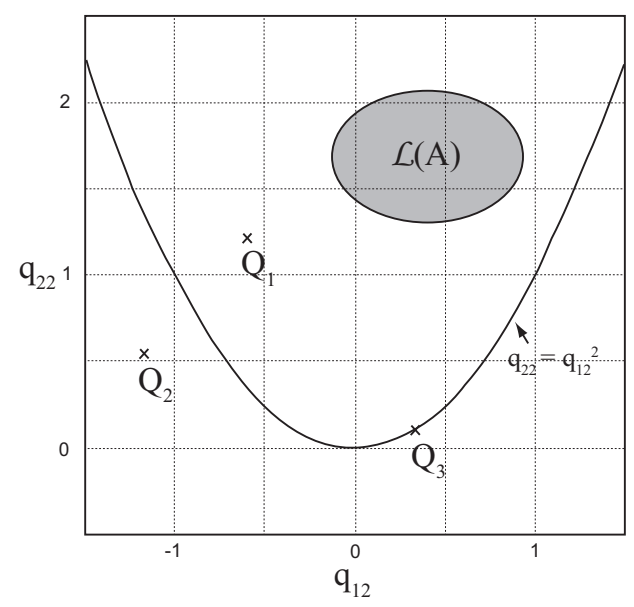

Fig. 2. Graphical representations of symmetric matrices. 
A necessary condition for the existence of a joint quadratic Lyapunov function $P \in \mathcal{P}_{2}(\mathbb{R})$ for the pairs $\left(A_{1}, \Omega_{1}\right)$ and $\left(A_{2}, \mathbb{R}^{2}\right)$ is that there is no convex combination of $A_{1}$ and $A_{2}$, or of $A_{1}$ and $A_{2}^{-1}$, which has an eigenvector in $\Omega_{1}$ with non-negative eigenvalue. Furthermore, this condition is sufficient to ensure that, for any $x \in \Omega_{1}$ and any $y \in \mathbb{R}^{2}$, no separating tangential hyperplane as described by (2) exists between $\mathcal{L}\left(A_{1}, \Omega_{1}\right)$ and $\mathcal{L}\left(A_{2}, \mathbb{R}^{2}\right)$.

Refer to [13] for proof. A separating tangential hyperplane as described by (2), given a vector $x \in \Omega_{1}$ and a vector $y \in \mathbb{R}^{2}$, is depicted in Fig. 3 by Type (a). Note that the above condition is similar to the condition for CQLF existence stated in Theorem 1. Now the unstable vectors of the convex combinations of $A_{1}$ and $A_{2}$ and of $A_{1}$ and $A_{2}^{-1}$ play a role. The condition presented in Theorem 3 is necessary but not sufficient for determining joint QLF existence in some cases. Consider a vector $x \notin \Omega$ and assume that $x_{1} \neq x_{2}$. Any line $\left\{P \mid x^{T} P A x=0\right\}$ which is tangent to $\mathcal{L}(A)$ is not also tangent to $\mathcal{L}(A, \Omega)$. Thus, even if the condition presented in Theorem 3 is satisfied, it may still be possible to separate two disjoint sets $\mathcal{L}\left(A_{1}, \Omega_{1}\right)$ and $\mathcal{L}\left(A_{2}, \mathbb{R}^{2}\right)$ with a tangential hyperplane of an alternative form as depicted in Figs. 3 and 4 by Types (b) and (c).

\section{MAIN RESULT}

We now present a necessary and sufficient solution to the joint QLF problem under consideration. Denote by $A(\Omega)$ the image of the region $\Omega$ under that action of the matrix $A$.

Theorem 4: Let $A_{1}, A_{2} \in \mathbb{R}^{2 \times 2}$ be two Hurwitz matrices. Suppose that $\Omega_{1}$ is a closed double cone of the form (1). There exists a joint quadratic Lyapunov function $P \in \mathcal{P}_{2}(\mathbb{R})$ for the pairs $\left(A_{1}, \Omega_{1}\right)$ and $\left(A_{2}, \mathbb{R}^{2}\right)$ if and only if the following conditions are satisified:

(i) there is no convex combination of $A_{1}$ and $A_{2}$, or of $A_{1}$ and $A_{2}^{-1}$, which has an eigenvector in $\Omega_{1}$ with nonnegative eigenvalue;

(ii) there is no convex combination of $A_{1}^{-1}$ and $A_{2}$, or of $A_{1}^{-1}$ and $A_{2}^{-1}$, which has an eigenvector in $A_{1}\left(\Omega_{1}\right)$ with non-negative eigenvalue;

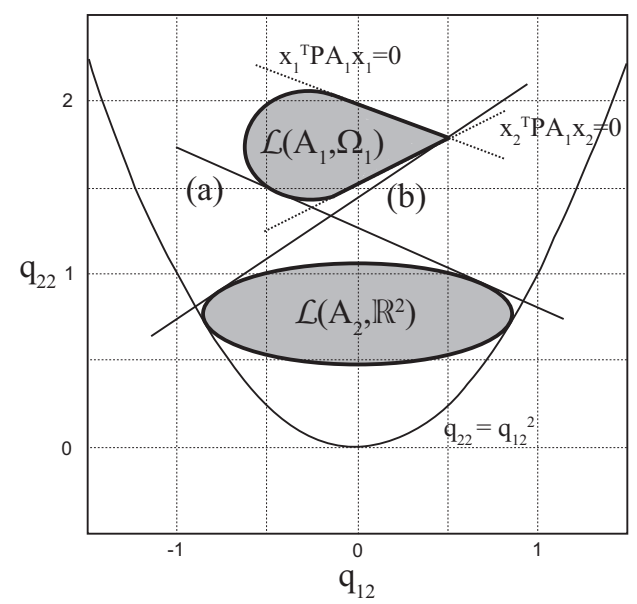

Fig. 3. Separating tangential hyperplanes: Types (a) and (b).

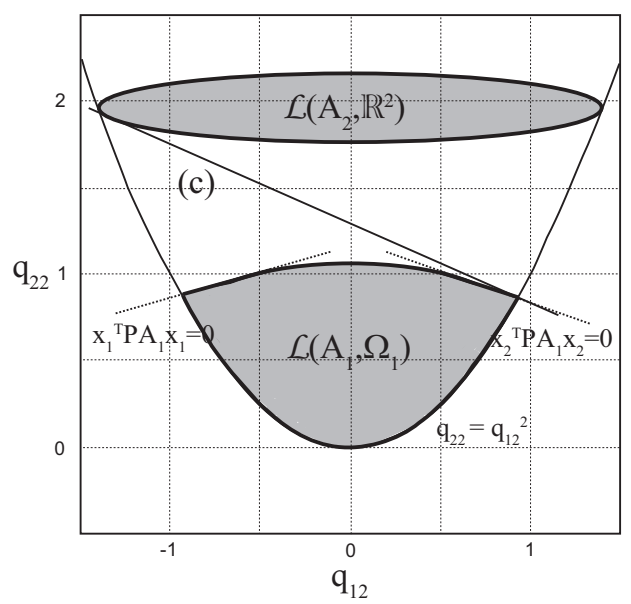

Fig. 4. Separating tangential hyperplanes: Type (c).

(iii) and there is no nonzero $y$ satisfying both equations

$$
\begin{aligned}
\left(a A_{1}+b A_{1}^{-1}+c A_{2}\right) y & =0, \\
a y y^{T}+b A_{1}^{-1} y y^{T}\left(A_{1}^{-1}\right)^{T} & =d_{1} x_{1} x_{1}^{T}+d_{2} x_{2} x_{2}^{T}
\end{aligned}
$$

for some non-negative coefficients $a, b, c, d_{1}, d_{2}$.

Refer to [13], [14] for proof. Note that (4) resembles the kinds of singularity conditions that arise for the CQLF problem in two dimensions. In fact, if $a=0$ or $b=0$ then (4) reduces to these conditions. Equation (5) is yet another condition of this form. However, rather than the singular eigenvectors being prohibited from $\Omega_{1}$, it is now required that symmetric matrices constructed from $y$ are prohibited from a region in matrix space spanned by $x_{1} x_{1}^{T}$ and $x_{2} x_{2}^{T}$. This latter, somewhat tedious condition, requires the construction of a multi-dimensional root locus. In the case of two-dimensional systems, one may circumvent this condition by representing the point in the $\left(q_{12}, q_{22}\right)$-plane at which the two hyperplanes $H_{1}:=\left\{P \mid x_{1}^{T} P A x_{1}=0\right\}$ and $H_{2}:=\left\{P \mid x_{2}^{T} P A x_{2}=0\right\}$ intersect with a very special dynamical system. (We shall denote this intersection point by $P_{0}$.) We will proceed to describe such a procedure in the next section.

Remark 5: If $P_{0} \notin \mathcal{P}_{2}(\mathbb{R})$, then conditions (i) and (ii) of Theorem 3 are necessary and sufficient for the existence of a joint QLF for the pairs $\left(A_{1}, \Omega_{1}\right)$ and $\left(A_{2}, \mathbb{R}^{2}\right)$. Condition (iii) is not required.

\section{CONIC INTERSECTIONS}

Recall that $P_{0}$ denotes the point where the hyperplanes $H_{1}$ and $H_{2}$ intersect. We associate with $P_{0}$ a matrix $Z$ such that the function $x^{T} P_{0} x$ defines a weak QLF [6, Appendix A.3] for the dynamical system $\dot{x}=Z x$. In particular, we fix $P_{0} \in \mathcal{P}_{2}(\mathbb{R})$ and define $Z=\sigma P_{0}$, where

$$
\sigma=\left[\begin{array}{cc}
0 & -1 \\
1 & 0
\end{array}\right]
$$

Note that $Z$ has pure imaginary eigenvalues; hence for each $\epsilon>0$ the matrix $Z-\epsilon I_{2}$ is Hurwitz, where $I_{2}$ is the $2 \times 2$ identity matrix. Furthermore $Z^{T} P_{0}+P_{0} Z=0$ and therefore 
the Lyapunov set $\mathcal{L}\left(Z-\epsilon I_{2}\right)$ contains $P_{0}$ for every $\epsilon>0$. Since $Z$ is not Hurwitz and since $\mathcal{L}\left(Z-\epsilon I_{2}\right)$ decreases as $\epsilon \rightarrow 0$ it follows that

$$
\bigcap_{\epsilon>0} \mathcal{L}\left(Z-\epsilon I_{2}\right)=\left\{k P_{0} \mid k>0\right\} .
$$

Thus, informally, $Z$ is the matrix whose "Lyapunov set" is just the intersection point $P_{0}$ as shown in Fig. 5.

A description for a separating tangential hyperplane of Type (b) as shown in Figs. 3 and 5 can be composed in the manner of (2) by equating oppositely oriented normal vectors as follows. Let $A_{2} \in \mathbb{R}^{2 \times 2}$ be a Hurwitz matrix and $l$ be a scalar. There are nonzero vectors $y, z \in \mathbb{R}^{2}$ satisfying the equation

$$
Z z z^{T}+z z^{T} Z^{T}=-l^{2}\left(A_{2} y y^{T}+y y^{T} A_{2}^{T}\right)
$$

if and only if $P_{0} \notin \mathcal{L}\left(A_{2}\right)$. To see this, note that if $P_{0} \in$ $\mathcal{L}\left(A_{2}\right)$ then $A_{2}^{T} P_{0}+P_{0} A_{2}<0$, however $Z^{T} P_{0}+P_{0} Z=0$ and so a solution to (6) cannot exist. If $P_{0} \notin \mathcal{L}\left(A_{2}\right)$ then, via application of Lemma 2, either

$$
\begin{aligned}
& z=s_{1} y \text { and } Z z=-\left(\frac{l^{2}}{s_{1}}\right) A_{2} y, \text { or } \\
& z=s_{2} A_{2} y \text { and } Z z=-\left(\frac{l^{2}}{s_{2}}\right) y
\end{aligned}
$$

for some real scalar $s_{1}$ or $s_{2}$, respectively. Suppose that $P_{0}$ is not in the closure of $\mathcal{L}\left(A_{2}\right)$. From (7), $\left[A_{2}^{-1} Z+\left(\frac{l}{s_{1}}\right)^{2} I\right] z=0$ which means that if (7) holds then $A_{2}^{-1} Z$ has two negative, real eigenvalues. From (8), $\left[A_{2} Z+\left(\frac{l}{s_{2}}\right)^{2} I\right] z=0$ which means that if (8) holds then $A_{2} Z$ has two negative, real eigenvalues. For future reference, we shall call the two (independent) eigenvectors of the matrix with positive, real eigenvalues $z_{3}$ and $z_{4}$ and call the eigenvectors of the matrix with negative, real eigenvalues $\tilde{z}_{3}$ and $\tilde{z}_{4}$. We also want to define a further four vectors $z_{1}, z_{2}, \tilde{z}_{1}$ and $\tilde{z}_{2}$. Let $z_{1}$ and $z_{2}$ be the eigenvectors of either the matrix $A_{1}^{-1} Z$ or $A_{1} Z$ which has positive, real eigenvalues and let $\tilde{z}_{1}$ and $\tilde{z}_{2}$ be the eigenvectors of the matrix which has negative, real eigenvalues.

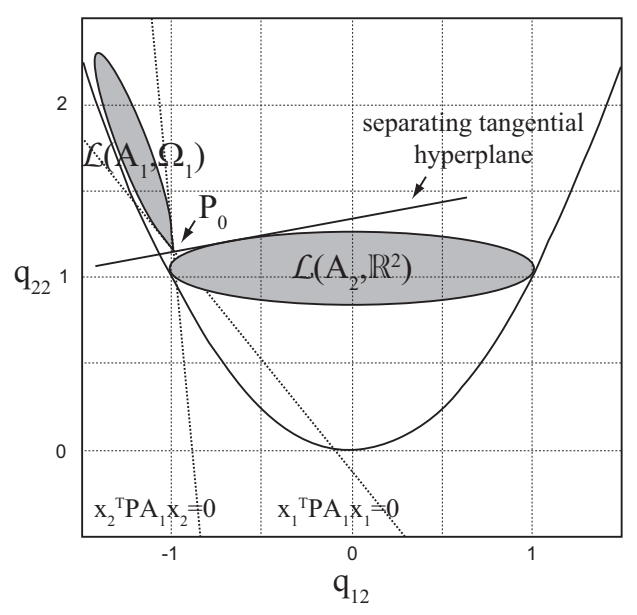

Fig. 5. Example.

\section{A. An Alternative to Condition (iii)}

As mentioned above, the matrix $Z$ has an imaginary spectrum and hence is not Hurwitz; therefore, the matrix $P Z+Z^{T} P$ cannot be negative definite for any $P \in \mathcal{P}_{2}(\mathbb{R})$. However, given a set of vectors $z_{1}, \ldots, z_{k} \in \mathbb{R}^{2}$, it may be possible to find a $P \in \mathcal{P}_{2}(\mathbb{R})$ such that $z_{j}^{T}\left(P Z+Z^{T} P\right) z_{j}<$ 0 for all $j=1, \ldots, k$. The next result provides a necessary and sufficient condition for this to occur. The development of an alternative solution to our joint QLF existence problem will require only four vectors, however we state the next result in full generality.

Before stating the result, two more definitions are provided. Given a vector $z \in \mathbb{R}^{2}$, there is a unique positive number $\alpha$ and a vector $\tilde{z} \in \mathbb{R}^{2}$ such that

$$
\tilde{z} \tilde{z}^{T}=\alpha P_{0}^{-1}-z z^{T}
$$

(note that $\tilde{z}$ is unique only up to sign). Next, let $L_{1}$ and $L_{2}$ be two finite collections of lines through the origin in $\mathbb{R}^{2}$. We assume all lines are distinct. Suppose there are $k$ lines in $L_{1}$, then these lines divide the plane into $2 k$ disjoint regions, each of them a pointed cone with vertex at the origin. These $2 k$ regions can be combined into $k$ double cones by taking the union of each region with its reflection in the origin. Then the collections $L_{1}$ and $L_{2}$ are defined to be disjoint if $L_{2}$ lies inside exactly one of these double cones.

Theorem 6: Let $z_{1}, \ldots, z_{k} \in \mathbb{R}^{2}$. Let $L$ be the collection of lines through the origin parallel to the vectors $z_{1}, \ldots, z_{k} \in$ $\mathbb{R}^{2}$, and let $\tilde{L}$ be the collection of lines through the origin parallel to the vectors $\tilde{z}_{1}, \ldots, \tilde{z}_{k}$, where $\tilde{z}_{j}$ is defined by (9) for each $j=1, \ldots, k$. Then there is a positive definite matrix $P \in \mathcal{P}_{2}(\mathbb{R})$ such that $z_{j}^{T}\left(P Z+Z^{T} P\right) z_{j}<0$ for all $j=1, \ldots, k$ if and only if $L$ and $\tilde{L}$ are disjoint.

Refer to [13] for proof. We now present an alternative solution to our joint QLF existence problem that circumvents Condition (iii) of Theorem 4. Again, refer to [13] for proof.

Theorem 7: Let $A_{1}, A_{2} \in \mathbb{R}^{2 \times 2}$ be two Hurwitz matrices. Suppose that $\Omega_{1}$ is a closed double cone of the form (1). Define $P_{0}, Z, z_{1}, \ldots, z_{4}$ and $\tilde{z}_{1}, \ldots, \tilde{z}_{4}$ as above. Let $L$ be the collection of lines through the origin parallel to the vectors $z_{1}, \ldots, z_{4} \in \mathbb{R}^{2}$, and let $\tilde{L}$ be the collection of lines through the origin parallel to the vectors $\tilde{z}_{1}, \ldots, \tilde{z}_{4}$. Consider the following conditions:

(i) There is no convex combination of $A_{1}$ and $A_{2}$, or of $A_{1}$ and $A_{2}^{-1}$, which has an eigenvector in $\Omega_{1}$ with nonnegative eigenvalue.

(ii) There is no convex combination of $A_{1}^{-1}$ and $A_{2}$, or of $A_{1}^{-1}$ and $A_{2}^{-1}$, which has an eigenvector in $A_{1}\left(\Omega_{1}\right)$ with non-negative eigenvalue.

(iii) $P_{0} \in \mathcal{P}_{2}(\mathbb{R}) \backslash \mathcal{L}\left(A_{2}\right)$ and the collections of lines $L$ and $\tilde{L}$ are disjoint.

If $P_{0} \notin \mathcal{P}_{2}(\mathbb{R})$, or if $P_{0} \in \mathcal{L}\left(A_{2}\right)$, then conditions (i) and (ii) are necessary and sufficient for the existence of a joint QLF for the pairs $\left(A_{1}, \Omega_{1}\right)$ and $\left(A_{2}, \mathbb{R}^{2}\right)$. If $P_{0} \in \mathcal{P}_{2}(\mathbb{R}) \backslash \mathcal{L}\left(A_{2}\right)$, and if $P_{0}$ is not in the closure of $\mathcal{L}\left(A_{2}\right)$, then conditions (i), (ii) and (iii) are necessary and sufficient for the existence of a joint QLF for the pairs $\left(A_{1}, \Omega_{1}\right)$ and $\left(A_{2}, \mathbb{R}^{2}\right)$. 


\section{EXAMPLE}

We include a simple example. Suppose that

$$
A_{1}=\left(\begin{array}{cc}
12 & -20 \\
12 & -17
\end{array}\right), A_{2}=\left(\begin{array}{cc}
-0.99 & -1 \\
-0.97 & -0.99
\end{array}\right)
$$

and that the vectors

$$
x_{1}=\left(\begin{array}{c}
0.1 \\
1
\end{array}\right), x_{2}=\left(\begin{array}{c}
1 \\
0.1
\end{array}\right)
$$

define the region $\Omega_{1}$. Solving the pair of linear equations $x_{1}^{T} P A_{1} x_{1}=0$ and $x_{2}^{T} P A_{1} x_{2}=0$ gives $P_{0}$ as follows:

$$
P_{0}=\left(\begin{array}{cc}
1 & -0.990574337 \\
-0.990574337 & 1.15872816
\end{array}\right) \text {. }
$$

Note that $P_{0} \in \mathcal{P}_{2}(\mathbb{R}) \backslash \mathcal{L}\left(A_{2}\right)$ and that $P_{0}$ is not in the closure of $\mathcal{L}\left(A_{2}\right)$. There exists no convex combination of $A_{1}$ and $A_{2}$, or of $A_{1}$ and $A_{2}^{-1}$, which has a nonnegative eigenvalue with associated eigenvector in $\Omega_{1}$. The condition in Theorem 3 is satisfied, meaning that a separating tangential hyperplane of Type (a) between the sets $\mathcal{L}\left(A_{1}, \Omega_{1}\right)$ and $\mathcal{L}\left(A_{2}, \mathbb{R}^{2}\right)$ cannot exist. It might still be possible that a tangential hyperplane of Type (b) and/or (c) separates the sets though.

The matrix $A_{1}^{-1} Z$ has two positive, real eigenvalues and so we label the eigenvectors of this matrix (or multiples of the eigenvectors of this matrix) $z_{1}$ and $z_{2}$. Since $A_{1} Z$ has two negative, real eigenvalues, we name the eigenvectors of this matrix (or multiples of the eigenvectors of this matrix) $\tilde{z}_{1}$ and $\tilde{z}_{2}$. Thus, let

$$
z_{1}=\left(\begin{array}{c}
0.1 \\
1
\end{array}\right), z_{2}=\left(\begin{array}{c}
1 \\
0.1
\end{array}\right)
$$

and

$$
\tilde{z}_{1}=\left(\begin{array}{c}
-18.8 \\
-15.8
\end{array}\right), \tilde{z}_{2}=\left(\begin{array}{c}
10 \\
10.3
\end{array}\right) .
$$

The matrix $A_{2} Z$ has two positive, real eigenvalues and so we label the eigenvectors of this matrix (or multiples of the eigenvectors of this matrix) $z_{3}$ and $z_{4}$. Since $A_{2}^{-1} Z$ has two negative, real eigenvalues, we name the eigenvectors of this matrix (or multiples of the eigenvectors of this matrix) $\tilde{z}_{3}$ and $\tilde{z}_{4}$. Let

$$
z_{3}=\left(\begin{array}{l}
-0.73068 \\
-0.68272
\end{array}\right), z_{4}=\left(\begin{array}{l}
-0.71539 \\
-0.69873
\end{array}\right)
$$

and

$$
\tilde{z}_{3}=\left(\begin{array}{c}
-0.77764 \\
0.62871
\end{array}\right), \tilde{z}_{4}=\left(\begin{array}{c}
0.97451 \\
-0.22436
\end{array}\right) .
$$

The resulting collections of lines $L$ and $\tilde{L}$ through the origin parallel to the vectors $z_{1}, \ldots, z_{4}$ and $\tilde{z}_{1}, \ldots, \tilde{z}_{4}$, respectively, are shown in Fig. 6. The collections are not disjoint. Thus, a joint QLF for the pairs $\left(A_{1}, \Omega_{1}\right)$ and $\left(A_{2}, \mathbb{R}^{2}\right)$ does not exist (as illustrated in Fig. 5).

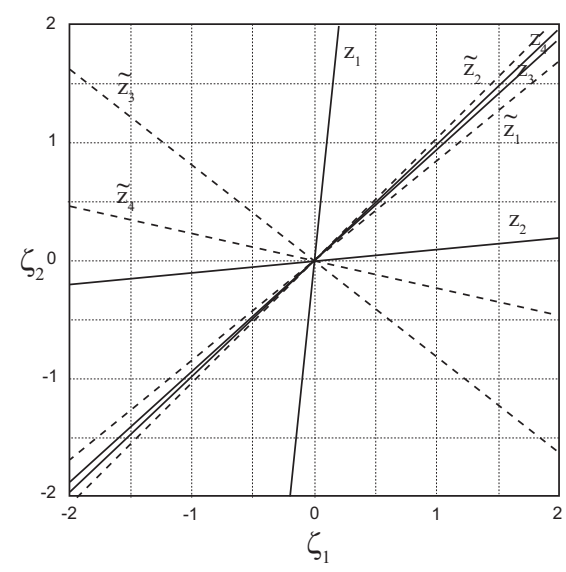

Fig. 6. Collections of lines, $L$ and $\tilde{L}$.

\section{CONCLUSIONS AND FUTURE WORK}

Algebraic necessary and sufficient conditions were obtained for determining joint QLF existence with respect to a two-dimensional, state-dependent switched system stability problem where a conic partition of the state space was considered. It is expected that some of the results presented in this paper will prove useful in extensions to higherdimensional systems.

\section{REFERENCES}

[1] H. Lin and P.J. Antsaklis, Stability and stabilizability of switched linear systems: a short survey of recent results, in Proc. IEEE International Symposium on Intelligent Control, Limassol, Cyprus, 2005, pp. 24-29.

[2] R.A. Decarlo, M.S. Branicky, S. Pettersson and B. Lennartson, Perspectives and results on the stability and stabilizability of hybrid systems, Proc. IEEE, vol. 88, no. 7, 2000, pp. 1069-1082.

[3] D. Liberzon and A.S. Morse, Basic problems in stability and design of switched systems, IEEE Control Syst. Mag., vol. 19, no. 5, 1999, pp. 59-70.

[4] M.S. Branicky, Multiple Lyapunov functions and other analysis tools for switched and hybrid systems, IEEE Trans. Automat. Control, vol. 43, no. 4, 1998, pp. 475-482.

[5] P. Peleties and R.A. Decarlo, Asymptotic stability of $m$-switched systems using Lyapunov-like functions, in Proc. Amer. Control Conf., Boston, MA, 1991, pp. 1679-1684.

[6] D. Liberzon, Switching in Systems and Control, Birkhäuser, Boston, MA; 2003.

[7] Z. Sun and S.S. Ge, Switched Linear Systems: Control and Design, Springer-Verlag, London, UK; 2005.

[8] R. Shorten, F. Wirth, O. Mason, K. Wulff and C. King, Stability criteria for switched and hybrid systems, SIAM Rev., vol. 49, no. 4, 2007, pp. 545-592.

[9] H.K. Khalil, Nonlinear Systems, Prentice Hall, Upper Saddle River, NJ; 1996.

[10] R.N. Shorten and K.S. Narendra, Necessary and sufficient conditions for the existence of a common quadratic Lyapunov function for a finite number of stable second order linear time-invariant systems, Internat. J. Adapt. Control Signal Process., vol. 16, 2002, pp. 709-728.

[11] S. Boyd and L. Vandenberghe, Convex Optimization, Cambridge University Press, Cambridge, UK; 2004.

[12] R. Shorten, K.S. Narendra and O. Mason, A result on common quadratic Lyapunov functions, IEEE Trans. Automat. Control, vol. 48, no. 1, 2003, pp. 110-113.

[13] W. Griggs, C. King, R. Shorten, O. Mason and K. Wulff, Preliminary results on the quadratic stability of state-dependent switching systems, submitted to Linear Algebra Appl.

[14] R. Shorten, C. King, K. Wulff and O. Mason, On quadratic stability of state-dependent planar switching systems, Tech. Rep. ISSN 14369915, TU Berlin, 2007. 